\title{
Robust Tree-Ring Detection
}

\author{
Mauricio Cerda ${ }^{1,3}$, Nancy Hitschfeld-Kahler ${ }^{1}$, and Domingo Mery ${ }^{2}$ \\ ${ }^{1}$ Department of Computer Science, University of Chile, \\ Blanco Encalada 2120, Santiago, Chile \\ mcerda, nancy@dcc.uchile.cl \\ ${ }^{2}$ Department of Computer Science, Pontificia Universidad Católica de Chile, \\ Av. Vicuña Mackenna 4860(143), Santiago, Chile \\ dmery@ing.puc.cl \\ ${ }^{3}$ INRIA-Loria Laboratory, Campus Scientifique 54506, \\ Vandoeuvre-lès-Nancy, France
}

\begin{abstract}
The study of tree-rings is a common task in dendrology. Usually the rings deliver information about the age of the tree, historic climate conditions and forest densities. Many different techniques exist to perform the tree-ring detection, but they commonly are semi-automatic. The main idea of this work is to propose an automatic process for the tree-ring detection and compare it with a manual detection made by an expert in dendrology. The proposed technique is based on a variant of the Generalized Hough Transform (GHT) created using a very simple growing model of the tree. The presented automatic algorithm shows tolerance to textured and very noisy images, giving a good tree-ring recognition in most of the cases. In particular, it correctly detects the $80 \%$ of the tree-rings in our sample database.
\end{abstract}

Keywords: dendrology, tree-ring, hough transform.

\section{Introduction}

The tree-rings or annual growth rings are formed in response to seasonal changes. Generally, a tree-ring is composed by two growth zones. In the first part of the growing season, thin-walled cells of large radial diameters are produced (earlywood), while towards the end of the season thick-walled cells of smaller diameter appears (latewood), resulting in a sharp disjunction between growth rings (see Fig. 11).

Analysis of tree-rings from cross-sections of the tree (called stem analysis) plays a main role in assessing growth response of trees to environmental factors. Furthermore, stem analysis is used to develop tree growth models to make yield and stand tables, and to reconstruct the entire historical growth record. Hence it has applicability in dendrochronological analysis 11. The tree-ring analysis is usually made recording the ring-width of four or eight directions on a wood disc, however in some applications it is necessary to record the entire growth ring [1], achieving a better estimation of ring areas.

${ }^{1}$ Study of woody plants such as shrubs and lianas.

D. Mery and L. Rueda (Eds.): PSIVT 2007, LNCS 4872, pp. 575-585 2007.

(C) Springer-Verlag Berlin Heidelberg 2007 
The automatization of the tree-ring recognition process is important because it could make more comparable and reproducible results, currently manually performed by experts. Additionally, an automatic algorithm could reduce the time required to perform the analysis. The automatization of the tree-ring recognition process requires of image analysis, but this is a tough task, because of a wood disc image contains a high level of noise. The noise of the wood disc images comes mainly from the texture and imperfections of the wood, and the acquisition process itself. Another problem is the difficulty to express the rings properties in any detection algorithm as constraints or desirable properties.

Each tree-ring can be approximated by using a polygon (closed polyline). The most obvious property of a tree-ring is that the polygon that represents it must have empty intersections with the polygons that represent the other rings. In addition, each polygon must contain the center of the wood disc (position of the pith 2). Some of the not obvious properties are that each ring is located at the transition dark-to-light in the latewood sector taking as reference point the pith position of the tree (see Fig. 10) and following the radial growth direction [1, and also, that the shape of one ring constrains the shape of the others. In Fig. 1] the shape similarity of close rings can be observed. The idea of this work is to propose a simple way to include those restrictions in the detection process in order to build an automatic algorithm for tree-ring detection.

In Section 2, we give an overview of different existing approaches for the treering detection and some other techniques that could be applied to this problem. The proposed algorithm is detailed in Section 3 and the results are presented in Section 4. Finally, the conclusions of our work are presented in Section 5.

\section{Overview}

From all the techniques used and proposed for the tree-ring detection problem, a simple classification can be made: techniques based on local features, techniques based on global models and other techniques. In the following section a discussion on the effectiveness of each technique is presented.

\subsection{Local Features Techniques}

The work of Conner 22 proposes a modified version of Canny edge detector [3] with a preferred edge orientation for each region of interest and a suppression of any edge that is not coming from a transition from latewood to earlywood. The inherent problem of this scheme is that assumes one edge orientation for a certain region of interest. The most interesting idea of the work of Conner [2] is the restriction imposed to the allowable edge orientations, but the main problem is that the restriction is fixed to one value for each region of interest.

Laggoune et al. 4] propose a different edge model that could handle noisy edges as the ones present in wood disc images. This approach is still strongly

${ }^{2}$ The oldest part of the tree, at its center, is called the pith. 


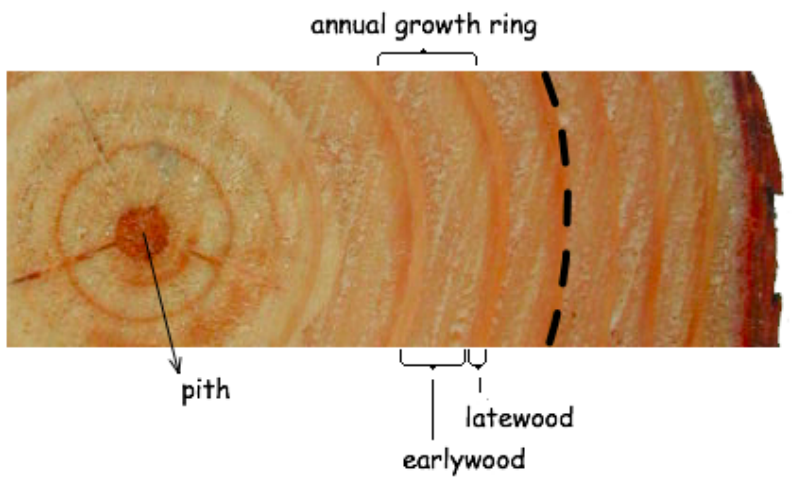

Fig. 1. The image shows a cross-cut from a Radiata pine trunk. The lighter zone indicates the earlywood, and the darker one the latewood. The abrupt edge of the latewood indicates the end of the growth season. The dotted line represents one possible approximate ring representation.

dependent on the kind of edge model assumed, then it does not always work. So it is possible to find wood disc images where in some parts, the Canny edge detector works better than noisy edge models as the one described in [4, and viceversa. Again, inherent to the local nature of this technique, there is no warranty that the output will be a closed shape for each tree-ring.

\subsection{Global Model Techniques}

One of the most simple techniques for template matching is the Hough Transform. In [5] a good review of this topic is given. The main restriction of the Hough Transform is that a certain shape must be usually assumed, for example a circle or an ellipse, and this delivers acceptable solutions only in a very reduced number of cases. In the other hand, a detection based on non-analytical shapes, as could be the output of a Generalized Hough Transform (GHT) 56] can not be used directly in the tree-rings problem. The GHT must be adapted first to the special characteristics of the tree-rings problem, as an example, there is no a priori ring shape to look for. Other techniques such as LevelSets [7, are not well suited to include restrictions specific to this problem, at least not in a simple way.

\subsection{Other Techniques}

The mentioned algorithms are mostly based on common techniques used in image processing, but a family of algorithms taking advantage of biologicalmorphological properties has also been developed. In order to understand the nature of some of these algorithms it is important to realize that the most common objective is not to detect each full ring, but other characteristics such as the number of rings, the area of the rings, and the mean ring width.

The work of Georg Von Arx et al. 8 presents an automatic technique to determine the mentioned characteristics and some additional ones. The authors 
use a high resolution image of a prepared sample where the wood cells can be visualized and appear in a different color. Using this image as input, the next step is to morphologically classify the cells according to the tree species in order to identify the ring zones. This work gives the idea that the input to detect the rings can be greatly improved (coloring cells) even before any process is applied, and it takes into account that the algorithm must make adjustments depending on the species. For the problem of recognizing the full shape of each ring, this technique does not deliver a good solution because it can not guarantee closed shapes and the rings can intersect each other.

In the work of Soille et al. 9] another approach is presented to compute the tree-ring area. The authors use different morphological operators and thresholding values to identify the ring zones and combine this information with the gradient of the image. The morphological filters are supposed to repair failures in the rings. The authors discuss the problem of too close rings and indicate that in some cases the approach does not deliver closed shapes.

Table 1. Comparison between the different existing algorithms

\begin{tabular}{llccc}
\hline Technique & Authors & \multicolumn{3}{c}{ Filter noise Impose shape Overlap rings } \\
\hline Local & -Conner [2] & No & No & Yes \\
& -Laggoune et al. [4] & Yes & No & Yes \\
\hline Global & -Hough Transform [5] & Yes & Yes & No \\
& -Generalized Hough Transform [6] & Yes & No & No \\
& -Level Sets [7] & Yes & - & - \\
\hline Others & -Georg Von Arx et al. [8] & No & No & No \\
& -Soille et al. [9] & No & No & No \\
\hline
\end{tabular}

For the tree-ring detection process, most of the previous techniques have been already tested on wood discs. Table 1 shows a comparison among them that takes into account desirable properties that a good recognition method should have. Since most of the reviewed techniques use only local or close to local information [24819], those techniques do not allow a proper recognition of the rings. A proper recognition method should consider global restrictions such as the similarity of the close rings and the influence of the shape of each ring on the neighboring ones, among others. As shown in Table 1, GHT global model shows more attractive characteristics but it must be adapted to the problem. We claim than our top-down GHT-based approach, described in the next section, is more similar to what the expert is implicitly doing in the manual processing and because of that, closer to what we have evaluated as a better technique than the known ones.

\section{Proposed Algorithm}

The proposed technique requires two parameters for each image, to compute the full ring set. These two parameters are the location of the center of the wood disc 


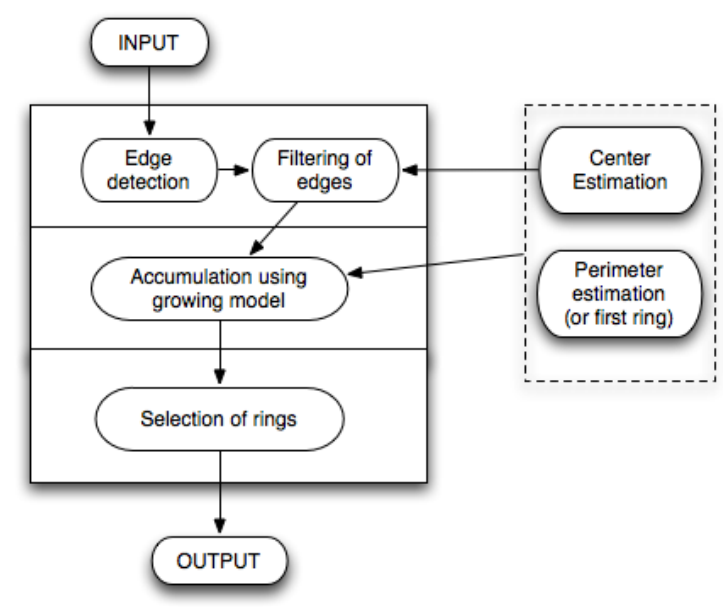

Fig. 2. Block diagram of the complete algorithm

image or pith $(C)$ and a polygon (convex or not) that represents the perimeter of the trunk $(P)$. Both parameters can be computed in different ways or even manually to have a semi-automatic algorithm. The description of the automatic procedure to compute $C$ and $P$ is explained in detail in Sections 3.2 and 3.3, respectively.

\subsection{Main Algorithm}

Fig. 2 shows a general diagram of the algorithm and Fig. 3 shows the algorithm applied to a wood disc image. The algorithm consists of three steps: Filtering (Fig. 3(b) and (c)), Accumulation (Fig. 3(d)) and Selection of the rings (Fig. 3(e)).

Filtering. The image is firstly transformed to the HSV color space, taking just the saturation component because this is the most representative value for the rings in the examined data. After this transformation, the Canny edge detection algorithm [3] is applied (any other gradient based technique could also be used). Then, for any point $Q$ detected as belonging to an edge (edge point), the following angle is computed:

$$
\alpha=\arccos \left(\frac{\|Q-C\|\left\|\left.\nabla I\right|_{Q}\right\|}{\left.<Q-C,\left.\nabla I\right|_{Q}\right\rangle}\right)
$$

where $I$ is the image intensity at point $Q$. Using a threshold level for the angle $\alpha$, it is possible to keep only dark-to-light edges. Note that usually the Canny algorithm delivers two edges for the latewood zone. In order to smooth this last process, we have applied two different thresholding levels, and for this, a standard double threshold linking was used. The output of this part of the algorithm is a binary image of not necessary connected edges but mostly in the correct ring locations. Noise is not completely removed at this point. 


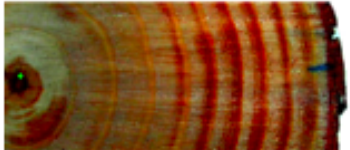

(a)

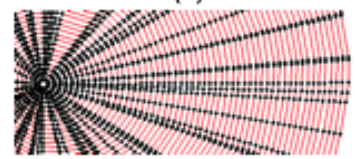

(d)

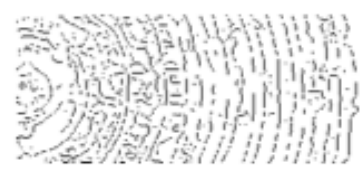

(b)

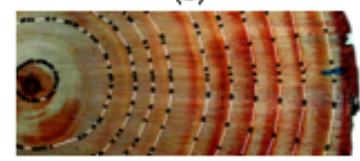

(e)

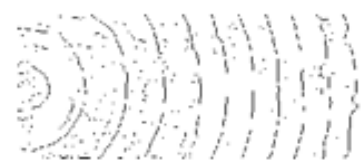

(c)

Fig. 3. Illustration of the proposed algorithm in each stage. (a) Sector of an input image. (b) Edges obtained after the Canny edge detector is applied to (a). (c) Edges obtained after the dark-to-light filtering was applied to (b). (d) All the possible selectable polygons computed from $P$ and $C$. (e) The selected rings from (d).

Accumulation. The growth model used to generate all the possible selectable polygons requires the tree trunk perimeter $P$, and the pith location $C$ of the wood disk. The following restriction on the shape of the polygon is then imposed: "Any polygon $R$ can be represented as a function of the tree trunk perimeter $P$ around the center of symmetry of the tree (pith) $C$ ", (see Fig. 4(a). We can represent this function by using the following expression:

$$
R_{i}\left(P_{i}, C, k\right)=C+k\left(C-P_{i}\right)
$$

where $R_{i}$ represents the $i-t h$ vertex in the selectable polygon of scale parameter $k$. $P_{i}$ is the $i-t h$ vertex of the tree trunk perimeter. The space of accumulation is $1 \mathrm{D}$ because is for the parameter $k$. The perimeter is not necessary a circle and $C$ is not necessary the center of the circle so, this scheme takes implicitly into account the normal asymmetries and the constraints of the rings. After the filtering, each point detected as belonging to an edge is assigned to the closest selectable polygon represented by a certain value of $k$. The output of this stage is an accumulator for $k$, that represents roughly the probability of each selectable polygon of being a tree-ring. In the simple case of an square image circumscribed about a circle $P$ with $C$ the center of that circle, each selectable polygon will be a circle of center $C, k$ will represent the normalized radius and the total number of selectable polygons will be at the most $N / 2$, where $N$ is the width of the image.

Selection of the Rings. The last stage of the algorithm takes the 1D accumulator and computes all the local maxima considering the maximum of three consecutive $k$ values. This way to compute the local maxima gives less false negative rings than taking five or more consecutive $\mathrm{k}$ values. Usually all the local maxima correspond to a ring, but it is necessary to fix a certain level of noise depending on the size of the image and on the size of the smaller tree-ring. For example in a $512 \times 512$ image, an accumulator with a value less than or equal to 10 for a certain polygon of scale parameter $\mathrm{k}$, is probably noise (see Fig. 4(b)]. 


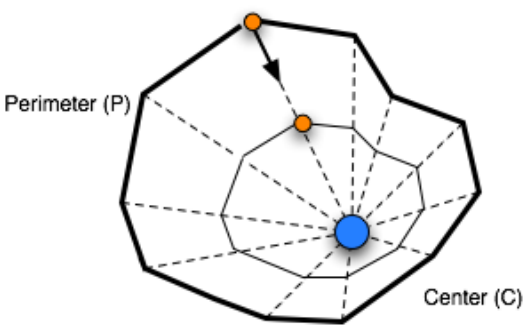

(a)

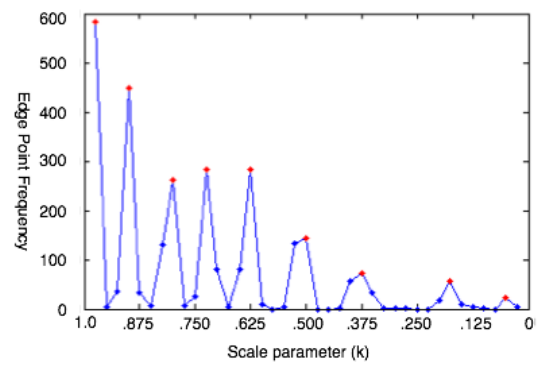

(b)

Fig. 4. (a) a ring is computed taking the $50 \%$ of P. (b) an accumulator for 40 possible scale changes $(\mathrm{k})$. Note that larger rings, more similar to the tree trunk perimeter, have a higher frequency value; this is because a larger ring is composed of more edge points.

\subsection{Center of the Wood Disc Image}

To estimate a point $\left(x_{0}, y_{0}\right)$, that represents the center of the wood disc image or pith, we can use a non-linear minimization process such as a gradient based method like the one mentioned in [4. By using this approach we find the point $\left(x_{0}, y_{0}\right)$ that minimizes the following objective function:

$$
J\left(x_{0}, y_{0}\right)=\sum_{i}\left(x_{i}-x_{0}\right)^{2}+\left(y_{i}-y_{0}\right)^{2}
$$

where $x_{i}$ and $y_{i}$ represent the coordinates of each point detected as belonging to an edge in the wood disc image. The minimization gives a point usually very close to the center, but not precise enough for the main algorithm. This is then corrected by using a common property observed in the sample database: the center is the darkest point around a certain distance from $\left(x_{0}, y_{0}\right)$. After testing this strategy in many images it is possible to fix the size of the neighborhood to be checked.

\subsection{Perimeter of the Wood Disc Image}

Several approaches were tested to select the most appropriate tree trunk perimeter approximation. The most simple one was to compute the convex hull of the edge image. The main problem with this approach is that it does not work well if the perimeter has large concavities or the shape of the tree bark is too different from the shape of the most external ring. The second approach was to use a Snake [5] algorithm but this also does not handle the concavities of the bark and it is quite more complex. Finally, the selected technique was to compute the perimeter using the convex hull of the edge image mostly because of the good results obtained in most of the wood disc images of the sample database and because of its simple implementation. 


\subsection{Implementation}

In the design of the implementation, one key factor was the performance of the algorithm. The most time consuming step in the presented algorithm is the accumulation stage. Considering an image of $N^{2}$ pixels, in the worst case all of them belong to an edge, the accumulator will have at the most $N$ possible values each one representing a possible ring and if each possible ring is composed of $M$ points ( $M$ segments), a brut force implementation will take time $O\left(N^{3} M\right)$ because for each pixel the closest possible ring must be calculated. A faster implementation is obtained by pre-computing the Voronoi diagram [10] of the vertices that form all the possible selectable rings and then iterating cell by cell of the diagram. This implementation takes time $O\left(N^{2}\right)$ to perform the accumulation. Note that the Voronoi diagram can be computed in time $O((N M) \log (N M))$ using the Quickhull algorithm. To give an idea of the final performance, the average time to process one wood disc (see Section 4), was $172 s$ : $46 \%$ of this time for the accumulation, $18 \%$ for the Voronoi computation, $12 \%$ for the center and filtering stage, and the rest was spent in reading and in the transformation operations such as RGB to HSV conversion.

\section{Results}

In this section we present and compare the ring detection results using the proposed automatic algorithm, the semi-automatic variant and a manual ring detection that we have called "real". The automatic version of the algorithm was applied by using the same parameters for the Canny edge detector and the double threshold linking in each one of the images. The semi-automatic technique was performed by asking the user for the perimeter, but the center point was still automatically obtained. All tests were performed using Matlab ${ }^{\circledR}$ Software [1],

Table 2. Table of results indicating the number of detected rings of each technique. TP: true positive rings, FN: false negative rings, $\hat{S_{n}}=\frac{T P}{T P+F N}$ : sensitivity.

\begin{tabular}{lcllllllllll}
\hline Images & Difficulty & \multicolumn{3}{c}{ Real } & \multicolumn{4}{c}{ Automatic } & \multicolumn{3}{c}{ Semi-automatic } \\
& $(1-10)$ & TP & FN & $\hat{S_{n}}$ & TP FN & $\hat{S_{n}}$ & TP FN & $\hat{S_{n}}$ \\
\hline Wood disc (base) & 3 & 9 & 0 & 1 & 9 & 0 & 1.00 & 9 & 0 & 1.00 \\
Wood disc 1 & 4 & 9 & 0 & 1 & 9 & 0 & 1.00 & 9 & 0 & 1.00 \\
Wood disc 2 & 5 & 11 & 0 & 1 & 10 & 1 & 0.90 & 10 & 1 & 0.90 \\
Wood disc 3 & 5 & 9 & 0 & 1 & 7 & 2 & 0.77 & 7 & 2 & 0.77 \\
Wood disc 4 & 6 & 12 & 0 & 1 & 7 & 5 & 0.58 & 10 & 2 & 0.83 \\
Wood disc 5 & 7 & 11 & 0 & 1 & 6 & 5 & 0.54 & 6 & 5 & 0.54 \\
Wood disc 6 & 7 & 10 & 0 & 1 & 10 & 1 & 0.90 & 10 & 0 & 1.00 \\
Wood disc 7 & 7 & 12 & 0 & 1 & 8 & 4 & 0.66 & 8 & 4 & 0.66 \\
Wood disc 8 & 7 & 9 & 0 & 1 & 8 & 1 & 0.88 & 8 & 1 & 0.88 \\
Wood disc 9 & 9 & 9 & 0 & 1 & 7 & 2 & 0.77 & 7 & 2 & 0.77 \\
\hline$<>$ & - & - & - & 1 & - & - & 0.80 & - & - & 0.85 \\
\hline
\end{tabular}


Table 3. Number of detected rings that mix two different manually detected rings

\begin{tabular}{lcccc}
\hline Images & Difficulty(1-10) & Real Automatic Semi-automatic \\
\hline Wood disc (base) & 3 & 0 & 0 & 0 \\
Wood disc 1 & 4 & 0 & 0 & 0 \\
Wood disc 2 & 5 & 0 & 1 & 1 \\
Wood disc 3 & 5 & 0 & 2 & 2 \\
Wood disc 4 & 6 & 0 & 2 & 2 \\
Wood disc 5 & 7 & 0 & 4 & 2 \\
Wood disc 6 & 7 & 0 & 1 & 0 \\
Wood disc 7 & 7 & 0 & 3 & 0 \\
Wood disc 8 & 7 & 0 & 0 & 0 \\
Wood disc 9 & 9 & 0 & 3 & 3 \\
\hline
\end{tabular}

with 10 color jpeg files of approximately 700 by 700 pixels each. The wood disk images were taken directly in the field with a Nikon Coolpix 885 camera. The chosen images include both, easy and difficult cases even for manual detection, and were selected for being representative of the main difficulties founded in the wood disc image database. The counting of the rings was performed automatically and the overlapping manually.

The results are summarized in Table 2 and Table 3. It can be seen that the proposed algorithm gives very good results. The automatic algorithm usually recognizes the same number of rings ( $\mathrm{TP}+$ overlaps) than the manual detection does, but sometimes, some of them are not true rings. This occurs when the detection algorithm mix two very close rings as shown in Table 3. This kind of problem in the automatic detection algorithm is produced when the bark of the tree does not give a good approximation of the first ring, as occurs in wood disc 5 and 7 . In this case, we recommend to use the semi-automatic algorithm because it usually improves the results. If the bark is too deformed is better to ask the user to directly indicate the first ring instead of the bark. It can be also seen in Table 2 and Table 3 that in the wood tree disc 9, the algorithm (automatic or semi-automatic) did not work well, but it was because this wood disc image contains a branch, situation that deforms the normal radial growth of the tree. The assumption that all the rings can be obtained by scaling the shape of the bark works well, when the bark is a good approximation of the first ring (usually this implies a thin bark) and the wood disc presents a close to regular growing (without branches).

\section{Conclusions}

In this work we present a robust automatic algorithm for tree-ring detection that works well in recognizing the rings of trees with normal or close to normal growing, tolerating false partial rings, textured zones and even additional lines. The proposed technique is composed of a filtering stage, followed by a voting for the re-scaling parameter of the perimeter. The new idea presented here is to take a 


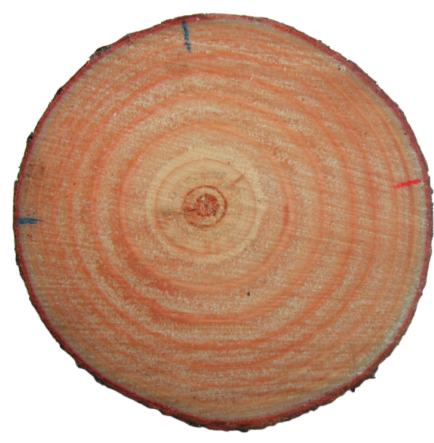

(a) Sample Tree 3.

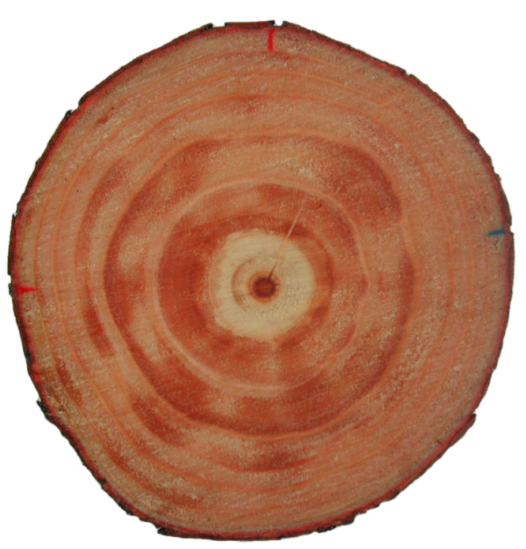

(c) Sample Tree 8.

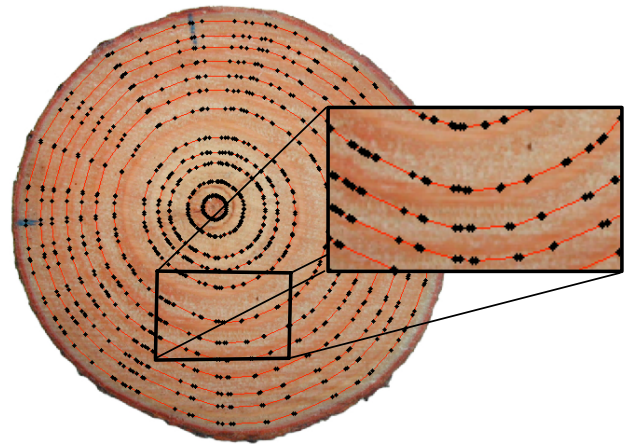

(b) Fully automatic recognition with the input figure 5(a) The detailed sector shows two overlapping rings.

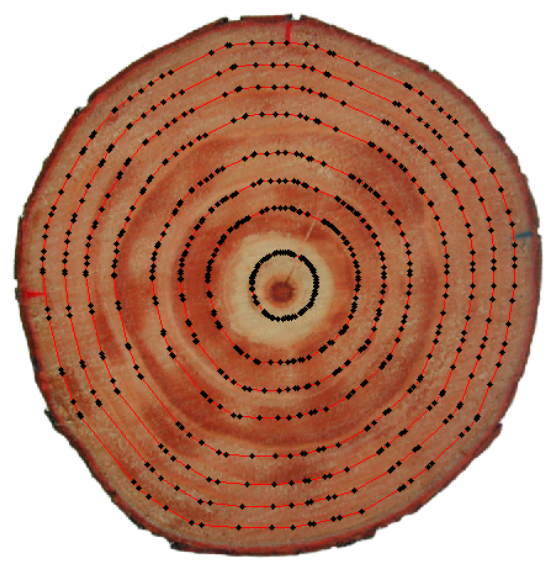

(d) Fully automatic recognition with the input figure 5(c).

Fig. 5. Two examples of input and result using the automatic algorithm

ring-prototype obtained from the bark of the wood tree image as input for this Hough-like-Transform without any previous assumption in the shape of the rings and to deform this ring-prototype using a very simple growing model of the tree. Future work includes (a) the use of different edge detection models more adapted to recognize noisy edges, (b) the use of a more accurate growing model for the tree rings and (c) the testing of the algorithm in different tree species.

\section{Acknowledgments}

Special thanks to Fernando Padilla from the Mathematical Modeling Center (CMM) of the Faculty of Physical Sciences and Mathematics (FCFM) of the University of Chile for the revision of the paper and valuable comments on how 
to improve it and for lending us the trunk image database and to Bernard Girau for useful comments about the paper. The authors acknowledge financial support to FONDECYT Chile - Project No. 1061227.

\section{References}

1. Forest, L., Padilla, F., Martínez, S., Demongeot, J., Martín, J.S.: Modelling of auxin transport affected by gravity and differential radial growth. Journal of Theoretical Biology 241, 241-251 (2006)

2. Conner, W.S., Schowengerdt, R.A.: Design of a computer vision based tree ring dating system. In: IEEE Southwest Symposium on Image Analysis and Interpretation, pp. 256-261 (1998)

3. Canny, J.: A computational approach to edge detection. IEEE Trans. Pattern Anal. Mach. Intell. 8, 679-698 (1986)

4. Laggoune, H., Sarifuddin, G.V.: Tree ring analysis. In: Canadian Conference on Electrical and Computer Engineering, pp. 1574-1577 (2005)

5. Nixon, M., Aguado, A.: Feature Extraction \& Image Processing. Elsevier, Amsterdam (2005)

6. Ballard, D.H.: Generalizing the hough transform to detect arbitrary shapes. Pattern Recognition 13(2), 111-122 (1981)

7. Sethian, J.A.: Level Set Methods and Fast Marching methods. Cambridge University Press, Cambridge (1999)

8. Arx, G.V., Dietz, H.: Automated image analysis of annual rings in the roots of perennial forbs. International Journal of Plant Sciences 166, 723-732 (2005)

9. Soille, P., Misson, L.: Tree ring area measurements using morphological image analysis. Can. J. For. Res. 31, 1074-1083 (2001)

10. Aurenhammer, F.: Voronoi diagrams - a survey of a fundamental geometric data structure. ACM Comput. Surv. 23, 345-405 (1991)

11. Mathworks: Image processing toolbox for use with Matlab: User Guide. The Mathworks Inc., Natick, MA, USA (2007) 